\title{
LA ARQUEOLOGÍA EN LOS GRANDES EVENTOS DE OCIO EDUCATIVO
}

Archaeology in big educative entertaiment events Francisco Javier Fernández de la Peña ${ }^{1}$ y Nuria Castañeda Clemente ${ }^{2}$. https://doi.org/10.15366/baexuam2020.14.006

\section{Resumen:}

Durante las ediciones de 2016 y 2017, Dibujantes de Arqueología participó en Juvenalia, feria emblemática del ocio educativo infantil y juvenil, desarrollando diferentes talleres arqueológicos. Este tipo de eventos masivos tienen ventajas e inconvenientes a los que se debe adaptar el diseño de las actividades pedagógicas. En este trabajo se analizan algunas de estas dificultades. También se analiza el impacto que estos talleres han tenido en redes sociales y medios de comunicación.

\section{Palabras clave:}

Juvenalia; ocio educativo; difusión científica; talleres arqueológicos; Dibujantes de Arqueología.

\begin{abstract}
:
Dibujantes de Arqueología took part in 2016 and 2017 Juvenalia editions, carrying out several archaeological workshops. Juvenalia is an emblematic fair of children's and youth entertainment. The design of pedagogic activities must be adapted to the advantages and disadvantages that this kind of massive events have. In this work, we analyse some of these problems. We also consider the impact of those workshops in social networks and mass media.
\end{abstract}

\section{Keywords:}

Juvenalia; educative entertainment; scientific diffusion; archaeological workshops; Dibujantes de Arqueología.

\section{Introducción}

La divulgación de la arqueología en España tiene ya un largo recorrido, desde las primeras experiencias erróneas y bien intencionadas del programa de RTVE "Misión rescate" entre los años 60 y 80 del siglo XX (Hernández 2019), hasta las iniciativas actuales de diversa naturaleza, que combinan las TIC y redes sociales (p. ej. Paleoaprende 2013; Proyecto Arqueo 2015). En cuanto a los proyectos educativopedagógicos, de integración de contenidos de arqueología en las aulas, se inician a finales de los 80 (p. ej. González-Marcén 1996, 1998), mediante charlas, dinamización

\footnotetext{
${ }^{1}$ DIBUJANTES de ARQUEOLOGÍA. Sitio Web: www.dibujantesdearqueologia.com. dibujantesarqueologia@gmail.com

${ }^{2}$ Investigadora postdoctoral programa Atracción del Talento Comunidad de Madrid. Dpto. de Prehistoria y Arqueología. Universidad Autónoma de Madrid.
} 
de yacimientos y museos, parques arqueológicos (p. ej. Pou et al. 1995; GonzálezMarcén et al. 1998; Castañeda y González 2000) y la modalidad que nos ocupa en este trabajo: los talleres (p. ej. Aragall 1987; Gil et al 1994; Jardón y Soler 1994).

El papel de la arqueología experimental y la experimentación o la denominada "experiencia del pasado" (Morgado y Baena 2011: 25; Baena et al. 2019:3), en pedagogía es fundamental. No solamente como contribución a la divulgación de contenidos científicos sobre el pasado, sino también como instrumento pedagógico de gran valor. La participación activa por parte de los agentes que reciben la información en forma de talleres, en los que se reproduce una parte o un proceso de trabajo preindustrial, contribuye a fijar la atención y crear recuerdos en conexión con emociones (Ausubel 2002; Gatt, 2003; Costa y Dorrío 2010; García-Marín y FernándezLópez 2020: 34; Pons y Serrano: 122). Por esa razón, los momentos lúdicos y de ocio, como las ferias (p. ej. Barrero et al. 2011), tienen un componente emocional para los más jóvenes que puede y debe aprovecharse para fijar conocimientos a través de las simulaciones, prácticas y experiencias (Llull 2011).

Dibujantes de Arqueología participó en las ediciones de 2016 y 2017 de Juvenalia, feria emblemática del ocio educativo infantil y juvenil, bajo el lema ;Vive la Arqueología! (Fig. 1). Sumando ambas convocatorias ${ }^{3}$, y durante once días, acudieron al evento un total aproximado de 178.000 personas.

En nuestro stand pudimos realizar una muestra de algunos de los talleres didácticos sobre Arqueología que habitualmente desarrollamos ${ }^{4}$ : la línea del tiempo, pintura rupestre, adorno personal en la prehistoria, cerámica prehistórica y la escritura en la Antigüedad.

\footnotetext{
3 Juvenalia 2016 se celebró del 6 al 11 de diciembre y recibió 84.766 visitantes (http://www.ifema.es/juvenalia_01/Prensa/NotasdePrensa/INS_086200). La edición de 2017 se llevó a cabo del 6 al 10 de diciembre $y$ fue visitada por 93.050 personas (http://www.ifema.es/juvenalia_01/Prensa/NotasdePrensa/INS_106791).

${ }^{4}$ Al margen de los talleres didácticos desarrollados para Juvenalia, los integrantes de Dibujantes de Arqueología han organizado actividades para diferentes colegios de la Comunidad de Madrid, para el Departamento de Difusión del Museo Arqueológico Nacional y han participado en varias ediciones de la Semana de la Ciencia de la Comunidad de Madrid, entre otros eventos.
} 


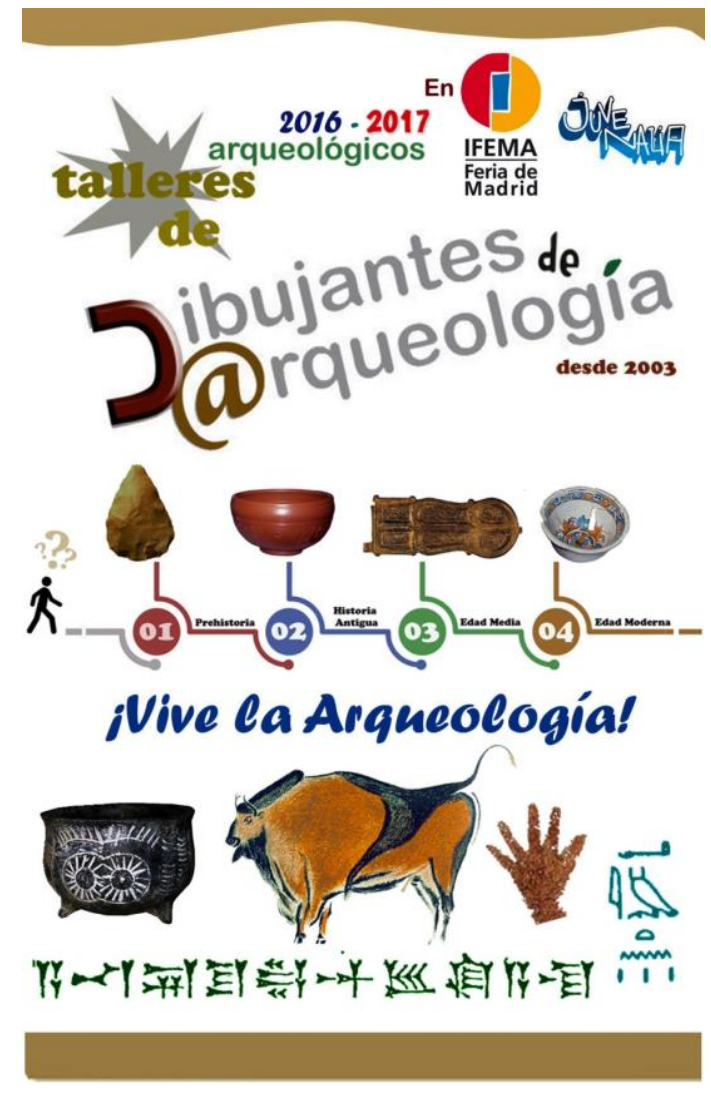

Figura 1: Cartel de los talleres arqueológicos de DIBUJANTES DE ARQUEOLOGÍA en Juvenalia 2016 y 2017.

\section{¿Qué es Juvenalia?}

Organizada por la Institución Ferial de Madrid (IFEMA) ${ }^{5}$, en el año 1980 se celebró el I Festival de la Infancia y la Juventud (Juvenalia 80). Durante once días (del 26 de diciembre al 5 de enero) y con casi un centenar de actividades, se pretendía con este evento un "acercamiento del joven a la sociedad real, al mundo de la producción, al funcionamiento del Estado, al avance tecnológico" (Tiza, Educación y Sociedad 1984: 49), "a través de la escuela, de los maestros como intermediarios privilegiados entre el joven y la sociedad" (Tiza, Educación y Sociedad 1984:3). Se intentaba, en definitiva, aproximar a los niños al mundo de los adultos con una serie de propuestas que "ponen a su alcance el mundo del arte, la cultura, la ciencia, la historia, la técnica y hasta la vida diaria del mundo en el que habitan (El País 1980)".

En sus primeras doce ediciones, Juvenalia se celebró de manera ininterrumpida en el Pabellón de Cristal de la Casa de Campo de Madrid durante las vacaciones de Navidad. En el año 1991, se trasladó a los nuevos recintos feriales del Campo de las Naciones, en el distrito de Barajas, lugar donde se sigue celebrando actualmente durante los días del puente de la Constitución.

En su primera etapa, desde Juvenalia 86 y durante varias ediciones, al margen de tener una mayor duración y celebrarse durante las vacaciones navideñas, la feria fue de

\footnotetext{
5 En la actualidad IFEMA es el primer organizador de ferias de España y el cuarto de Europa. Anualmente se celebran más de 80 ferias, con la participación de más de 32.000 empresas. Entre las ferias que organiza destacan: Fitur, Fruit Attraction, Arco o Intergift, entre otras.
} 
carácter gratuito, lo que permitió obtener las mayores cifras de visitantes. Por ejemplo, en 1987, se llegaron a contabilizar 509.000 personas (El País 1987).

Centrada cada edición en un tema de interés en particular (en 1988, en el mundo de la ciencia y la tecnología; en 1993, el deporte y la aventura; en 1998, en la industria del cine; en 1999, en Internet y las comunicaciones, etc.), el ámbito histórico y/o arqueológico también estuvo presente con mayor o menor protagonismo y desde distintos enfoques. En Juvenalia 1990, a modo de atracción, se realizó una réplica de una pirámide maya de $10 \mathrm{~m}$ de altura y $337 \mathrm{~m}^{2}$ de extensión, en cuyo interior se desarrollaba un circuito de aventuras con una base de operaciones arqueológicas incluida (Pozo 1990: 53). En Juvenalia 2000, convocada bajo el título de "Historias y Números"6, se recreaba para el visitante la España de Carlos V incluyendo la reproducción de una pequeña ciudad del siglo XVI, con sus plazas, corralas, talleres, callejuelas y coloridos mercadillos, para aproximar las costumbres de la vida cotidiana de la época y los hechos relevantes de la Historia de España (Galindo 2000).

Durante la segunda etapa, con su traslado en 1991 a la nueva zona del Campo de las Naciones, Juvenalia sufrió la cancelación de la edición de 2010 (EFE 2009). Los motivos fueron los recortes presupuestarios del gobierno regional de la Comunidad de Madrid y la creación en paralelo de un nuevo formato que se celebra desde 2011, teniendo como eje fundamental el concepto de ocio familiar: Dabadúm, salón del ocio infantil en familia.

Finalmente, en el año 2015, junto a Dabadúm, se recuperó de nuevo Juvenalia para crear una única gran convocatoria de ocio: Juvenalia-Dabadúm. Como novedades a destacar, se amplió la oferta de actividades hasta los 16 años y, sobre todo, se intentó dar un mayor protagonismo a la ciencia y al fomento de la investigación científica en España incluyendo los talleres y actividades de la Asociación Apadrina la Ciencia ${ }^{7}$. Por otro lado, en la edición de 2016, una de las principales novedades fue la introducción de la arqueología al público a través de distintos talleres.

Aunque en diferentes ocasiones Juvenalia ha sido criticada por su carácter consumista y comercial debido a la presencia de grandes empresas privadas y firmas comerciales, cabe subrayar la participación en diferentes ediciones de instituciones públicas de renombre tanto nacionales como internacionales como el Consejo Superior de Investigaciones Científicas (CSIC), el Museo de la Ciencia de Barcelona, la Ciudad de las Ciencias y la Industria de La Villette, de París, el Centro Pompidou (Atelier des Enfants) y la Facultad de Matemáticas de la Universidad de Milán, entre otros.

\section{Objetivos de los talleres arqueológicos}

El objetivo de los talleres de Dibujantes de Arqueología es trabajar conceptos curriculares adaptados a las edades de los niños participantes a través de la arqueología como hilo conductor. De esta manera, los alumnos no solo desarrollan capacidades manuales experimentando procesos de trabajo de la prehistoria y la arqueología, sino

\footnotetext{
${ }^{6}$ La segunda parte del título ("números") hace referencia a que el año 2000 fue declarado año mundial de las Matemáticas.

${ }^{7}$ https://idealia.wixsite.com/apadrina-la-ciencia/our_team
} 
que también relacionan contenidos de varias disciplinas académicas como las Matemáticas, las Ciencias Naturales, la Historia o el Lenguaje. Asimismo, estas actividades fomentan la curiosidad por conocer y entender otras culturas diferentes y la valoración del Patrimonio histórico-arqueológico.

Otro de los objetivos principales es la difusión de la Arqueología como ciencia que extrae sus conocimientos mediante métodos específicos y datos objetivos. A este respecto, la arqueología experimental juega un papel clave como fuente de datos de contraste de hipótesis (p. ej. Baena et al. 2019).

Todas las actividades se componen de una breve introducción teórica, la práctica de la actividad por parte de los participantes y el trabajo de los conceptos mediante fichas didácticas (Fig. 2), fomentando el aprendizaje significativo ${ }^{8}$ (Ausubel, 2002; Gatt 2003; Kim et al. 2018). La sensación de realizar tareas propias de otras épocas a las que no está acostumbrado suscita en el alumno una motivación que es imprescindible para fijar los conocimientos adquiridos (p. ej. Ausubel 2002; Shernoff y Hoogstra 2001; Carrillo et al. 2009; Kim et al. 2018). Por ello, estas actividades didácticas constituyen un recurso ideal para complementar el aprendizaje de cualquier asignatura.

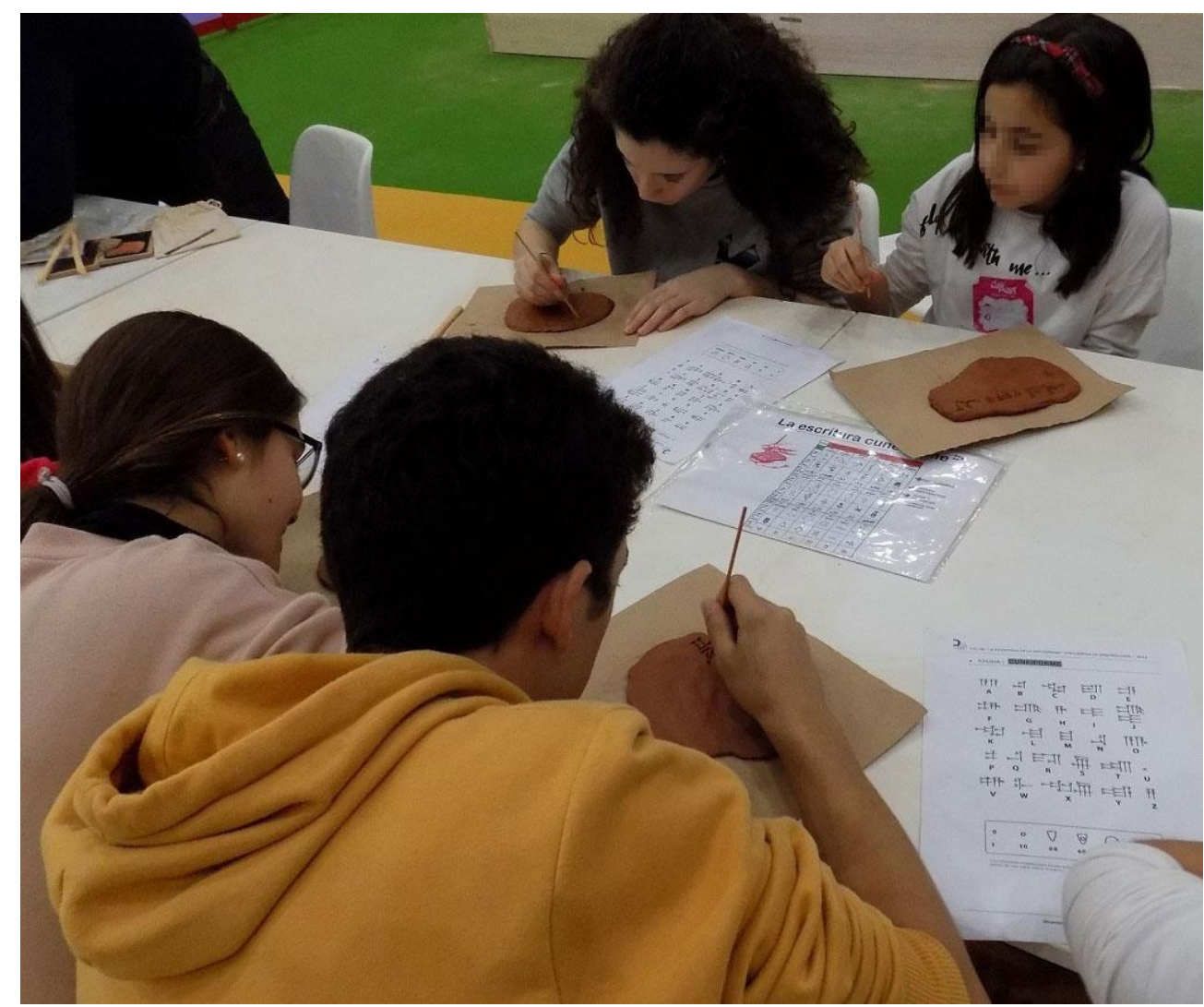

Figura 2: Actividades con fichas didácticas en el taller de "La escritura en la Antigüedad".

8 Según la teoría de David Ausubel, un aprendizaje es significativo cuando los contenidos son relacionados de modo no arbitrario y sustancial (no al pie de la letra) con lo que el alumno ya sabe (Ausubel et al., 1983:18). De este modo, en el proceso educativo, es importante considerar lo que el individuo ya sabe de tal manera que establezca una relación con aquello que debe aprender. En este tipo de aprendizaje un estudiante asocia la información nueva con la que ya posee; reajustando y reconstruyendo ambas informaciones en este proceso. 
Los ámbitos en los que solemos realizar los talleres didácticos suelen ser propicios a la reflexión, la explicación, el debate y el trabajo individual con fichas: talleres en colegios, asociaciones o eventos como la Semana de la Ciencia.

Sin embargo, realizar estos talleres en Juvenalia ha supuesto un esfuerzo de adaptación metodológica, ya que las condiciones antes descritas no son posibles. Los talleres que realizamos en estas pasadas ediciones de Juvenalia son excepcionales como excepcionales son las circunstancias que los caracterizan. Estas características son las siguientes: el exceso de estímulos, el ruido, la presencia de los padres, las diferentes edades y los límites de tiempo.

En este tipo de eventos masificados, los niños están sobreexcitados con multitud de estímulos a su alrededor, todos dirigidos a captar su atención. El evento está enfocado al ocio principalmente y aún es raro que una tarea reflexiva y sosegada se entienda como tal. Por eso, es importante que unos talleres de arqueología bien diseñados y dirigidos tengan su espacio en estos eventos.

Cuando los niños llegan a la actividad se encuentran eufóricos. Pueden venir de tirarse por un tobogán gigante, de jugar a videojuegos o de bajar de la tirolina de los bomberos. Esperan ese mismo nivel de adrenalina y, aunque arqueología y aventuras haya sido un binomio recurrente, como mucho, en un taller lo que podemos sugerir es misterio ante algo que ellos aún no conocen.

En nuestros talleres el único estímulo visual que había era la reproducción de un abrigo rupestre. Este elemento aislaba la zona de talleres de una parte del ruido que parece ser inherente a estos eventos: música procedente de varias fuentes simultáneas, locutores, gritos de júbilo, etc. Es decir, las explicaciones orales cuentan con otra dificultad añadida.

La presencia de los padres en los talleres es un factor difícil de controlar. Pero, aunque es obvio que los niños no actúan de la misma manera en presencia de los padres, en realidad lo que obtenemos es una radiografía de los diferentes estilos de educación. De ellos, la forma más perniciosa es la del progenitor que decide que aprender no es divertido, sobre todo si se trata de "contenidos no STEM".

En la sociedad actual, los niños crecen en un entorno en el que tanto el ocio como el aprendizaje son activos (p. ej. Kim et al. 2018). Sin embargo, la idea de que aprender cosas que se enseñan en el colegio, puede ser también una forma de ocio aún no está muy arraigada (European Commission 1995:2; Lull 2011; Aguilar et al. 2013). En general, las familias que acuden a Juvenalia dejan que sus hijos escojan qué actividades quieren realizar allí. Sin embargo, algunos padres sugieren o dirigen a sus hijos hacia otras actividades cuando se acercan a los stands de talleres de ciencia o culturales, como el nuestro, aunque los niños hayan manifestado su interés. En algunos casos, porque se pueden ensuciar, en otros porque ignoran en qué consiste la diversión, y en otros porque creen que es mejor aprovechar el tiempo en otros tipos de ocio más superficiales o vacíos.

\footnotetext{
${ }^{9}$ El término STEM es el acrónimo de los términos en inglés Science, Technology, Engineering and Mathematics (Ciencia, Tecnología, Ingeniería y Matemáticas).
} 
En otros casos, hay padres que no confían en las habilidades de sus hijos o creen que lo que nosotros les proponemos es demasiado difícil para ellos. Entonces, ellos insisten en pasar a hacer el taller con ellos o gritan y dan explicaciones desde la zona de espera, en el exterior del stand. Lógicamente, todo eso son interrupciones y dificultades que hay que sortear, ya que cuando se consigue obviarles, ese tipo de padres observa que los niños han conseguido hacer la actividad sin ellos, se asombran y también aprenden a confiar en las capacidades de sus hijos.

Ha sido muy interesante observar el comportamiento de algunos niños y niñas que son capaces de sosegarse, prestar atención y educar su paciencia al participar en nuestros talleres, ante el asombro de sus padres. En general, este tipo de actividades tranquilas no se fomentan. En esta sociedad en la que se promociona la recompensa inmediata, nadie quiere esperar (p. ej. Mischel et al. 1988; O’Brien et al. 2011; Aguilar et al. 2013: 197; Mr.Contraintuitivo 2019), ni los niños, ni sus padres que son los que esperan la cola de las actividades. Sentar a los niños en sillas y explicarles una actividad, después de haber esperado el turno, es todo un logro.

Limitar las edades de los talleres según su dificultad y el número de niños, es la estrategia ideal para enfrentarse a grupos de edad muy dispares. Desde los alumnos de ESO hasta los de infantil, todos se interesan en una u otra actividad. Sin embargo, hay que procurar adaptar la explicación y la actividad "en escalones" según el rango de edad para poder conseguir un nivel de dificultad adecuado que ayude a mantener la motivación y la concentración (Csikszentmihalyi 1997). También el aumento de la autonomía del niño en la actividad favorece la atención a diferentes edades de forma simultánea.

Por último, las actividades deben ser más cortas de lo habitual, pero no demasiado para no eliminar el contenido que es el objetivo de la realización de estos talleres. Sin embargo, por los factores que se han comentado anteriormente (sobreestimulación, ruido, padres que están esperando la cola en otra actividad), las explicaciones deben ser breves y personalizadas, así como la adaptación de las fichas a duraciones diferentes. Los talleres se convocaban a determinadas horas y tenían una duración mínima. A partir de esa duración, los niños podían quedarse a profundizar más en la actividad o bien podían dejarla si los padres les avisaban del comienzo de otra actividad. Algunos niños muy interesados decidían pasar el máximo tiempo posible en nuestros talleres.

\section{Descripción de los talleres arqueológicos}

En nuestra participación en las ediciones 2016 y 2017 de Juvenalia, hemos realizado los siguientes talleres: la línea del tiempo, pintura rupestre, adorno personal en la Prehistoria, cerámica prehistórica y la escritura en la Antigüedad (Fig. 3).

El taller "La línea del tiempo" solo se realizó en la edición de 2016. La actividad consistía en una breve explicación oral, apoyada por carteles y esquemas, seguida de la realización de un recorrido desde el Paleolítico hasta la Edad Moderna a través de diversos objetos cotidianos que los niños podían tocar. Para ello, se utilizaron reproducciones de piezas arqueológicas que reflejaba no solo los cambios tecnológicos a través del tiempo, sino también las diferentes expresiones culturales de las personas que los realizaban y los utilizaban. Durante este taller se fomenta el diálogo y la 
reflexión sobre la evolución tecnológica y las diferentes culturas que han habitado la Península Ibérica a través de los siglos. Dado que este era el taller menos activo por parte de los niños, se decidió no volver a realizarlo en la siguiente edición.

En el taller "Pintura rupestre" los niños realizan representaciones de los motivos propios del arte paleolítico y levantino. El taller comienza por una atención personalizada en la que cada niño recibe de forma individual, un equipo de protección contra las manchas, las herramientas y una breve explicación de la actividad, mostrándole ejemplos abundantes de representaciones rupestres. Para la realización de este taller se utilizó una recreación de un abrigo rupestre donde se realizaban las pinturas utilizando las técnicas y materiales propios de la época (p. ej. Chalmin et al. 2003), aprendiendo el valor de estas manifestaciones gráficas como expresión de unos de los pobladores más antiguos de la Península Ibérica. En esta actividad, se puso el énfasis en el conocimiento de los motivos menos conocidos de estas representaciones, así como el procedimiento para realizarlas y la información que obtenemos de la investigación sobre estas pinturas. Este fue uno de los talleres con más éxito de los que realizamos ya que es apto para todas las edades y porque los niños tienen pocas oportunidades de pintar en un soporte vertical de grandes dimensiones, que lo hacía muy gratificante. Además, pintar es uno de los recursos que más gustan a los niños.

El taller "Adorno personal en la Prehistoria" consistió en una introducción breve seguida de la elaboración de colgantes y cordelería con las técnicas utilizadas en el Paleolítico y Neolítico (p. ej. Mărgărit 2016). Mediante la perforación de conchas (cardium edule) y la realización de cuentas de collar en piedra (esteatita) los alumnos valoran el trabajo minucioso y delicado que se esconde tras objetos aparentemente humildes. Esta actividad sirve también para la reflexión sobre el hecho de adornarse en época prehistórica y en otras culturas. La actividad se completaba con fichas para colorear y completar sobre modelos de adorno personal en los períodos estudiados. Este taller solamente pudo realizarse en la edición de 2016, ya que requería demasiada atención personalizada con cada alumno para los niveles de sobre estimulación con los que acudían los niños. 

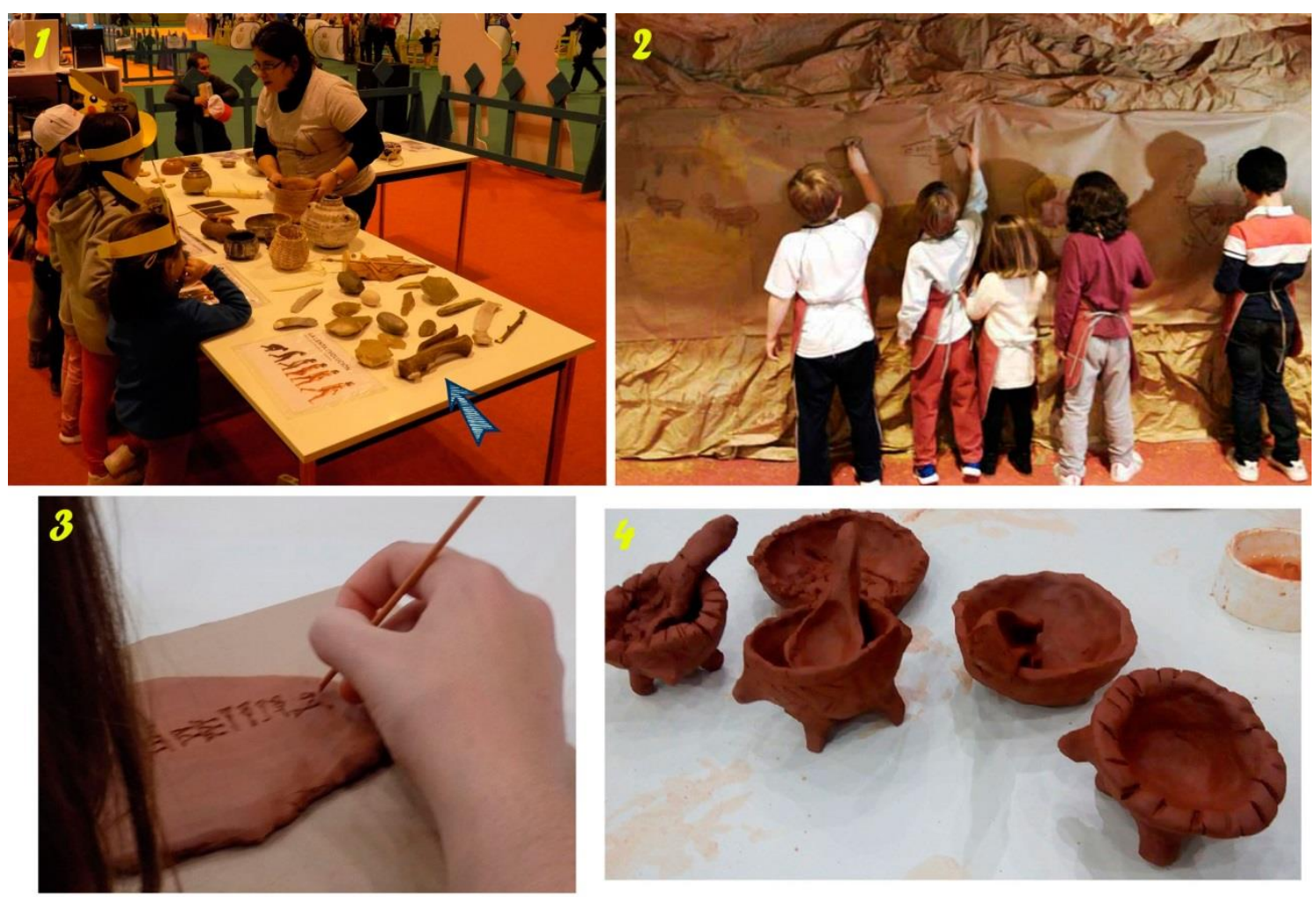

Figura 3: Imágenes de algunos de los talleres desarrollados en Juvenalia por Dibujantes de Arqueología: 1- "La línea del tiempo"; 2- "Pintura rupestre"; 3- "La escritura en la Antigüedad" y 4- "Cerámica prehistórica".

En el taller de "Cerámica prehistórica", tras una breve explicación de la actividad, los participantes examinan y tocan algunas reproducciones de cerámica prehistórica y después proceden a la fabricación de un pequeño cuenco de arcilla. En esta actividad se desarrolla la paciencia y la psicomotricidad fina. Además, la realización de un cuenco a mano mediante la técnica de "churros" con un material plástico sobre el que pueden corregirse los errores, ayuda a valorar el trabajo artesanal manual. Durante esta actividad se reflexiona sobre el aprovechamiento de los recursos naturales en relación con una de las aportaciones tecnológicas más importantes de la Historia de la Humanidad. Esta actividad tuvo un gran éxito en las dos ediciones, pese al miedo de los padres a las manchas de la arcilla. En la sociedad actual, una persona puede crecer sin apenas hacer cosas con las manos y ciertas capacidades pueden están perdiéndose (García-Marín y Fernández-López 2020:34). Quizás este sea uno de los motivos por los que los niños adoran trabajar con la arcilla, aparte de que sea un soporte que estimula la creatividad y la manipulación. Elaborar un cuenco con unas técnicas concretas, estimula la meticulosidad y también la creatividad al observar las posibilidades que esa técnica ofrece.

Por último, en "La escritura en la Antigüedad" los participantes escribieron su nombre y su edad en cuneiforme sobre una tablilla de arcilla y descifraron sencillos mensajes en jeroglífico egipcio, con ayuda de unas fichas elaboradas a tal fin. En este taller también se trabajó sobre las Matemáticas y las primeras expresiones numéricas, realizando operaciones aritméticas básicas como sumas y restas. Esta actividad nos introduce en el origen de la escritura a partir de los primeros pictogramas como avance de importancia crítica en la Historia. Este taller se introdujo en la edición de 2017, conscientes del reto que suponía. Se trata de una actividad apropiada a partir de los 8 años y tuvo un gran éxito en el rango de edad de 9 a 12 años. Los participantes se mostraban ajenos al 
tiempo empleado y a la dificultad de unos signos que les eran completamente extraños (Kim et al 2018). Niños con menor capacidad de atención y concentración respondieron de forma muy positiva ante este taller, por la excelente combinación entre la dificultad, el reto y la recompensa (Csikszentmihalyi 1997).

\section{Difusión de los talleres arqueológicos en las redes sociales}

Conscientes de la potencia de las redes sociales para difundir cualquier tipo de evento, durante los días de celebración de Juvenalia 2016 y 2017, estuvimos publicando (en ocasiones, en directo) diversas novedades sobre lo que allí estaba sucediendo.

Empleando diversos formatos (textos, imágenes y videos) y diferentes canales (Twitter, Facebook, Google Plus, YouTube y nuestro propio sitio web ${ }^{10}$ ), cabe destacar el alcance orgánico logrado especialmente en Facebook ${ }^{11}$, y particularmente con la publicación del video sobre el taller de "Pintura rupestre", que tuvo una gran interacción (sobre todo, entre mujeres de 35 a 44 años) y fue muy compartido (Fig. 4).

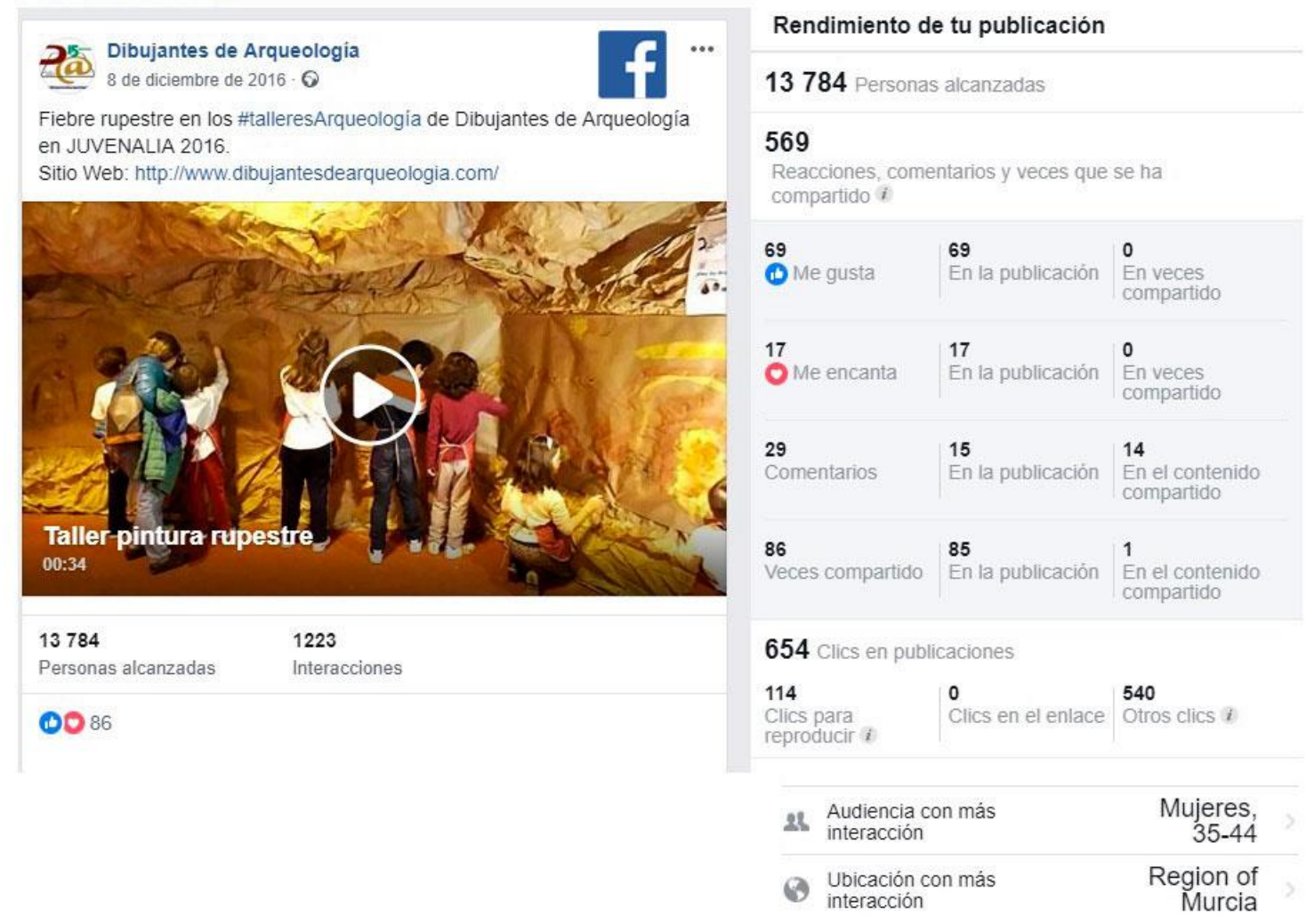

Figura 4: Datos sobre el alcance orgánico y rendimiento obtenido con el video del taller de "Pintura rupestre" publicado el 8 de diciembre de 2016 en el canal de Facebook de Dibujantes de Arqueología (2016).

Por otro lado, la magnitud del evento Juvenalia y la novedad de la arqueología entre las opciones de ocio infantil y juvenil, hizo que nuestros talleres también encontraran eco en los medios de comunicación (Fig. 5).

\footnotetext{
$10 \mathrm{https}: / /$ www.dibujantesdearqueologia.com/

${ }^{11} \mathrm{https}$ ://es-es.facebook.com/dibujantesdearqueologia/
} 


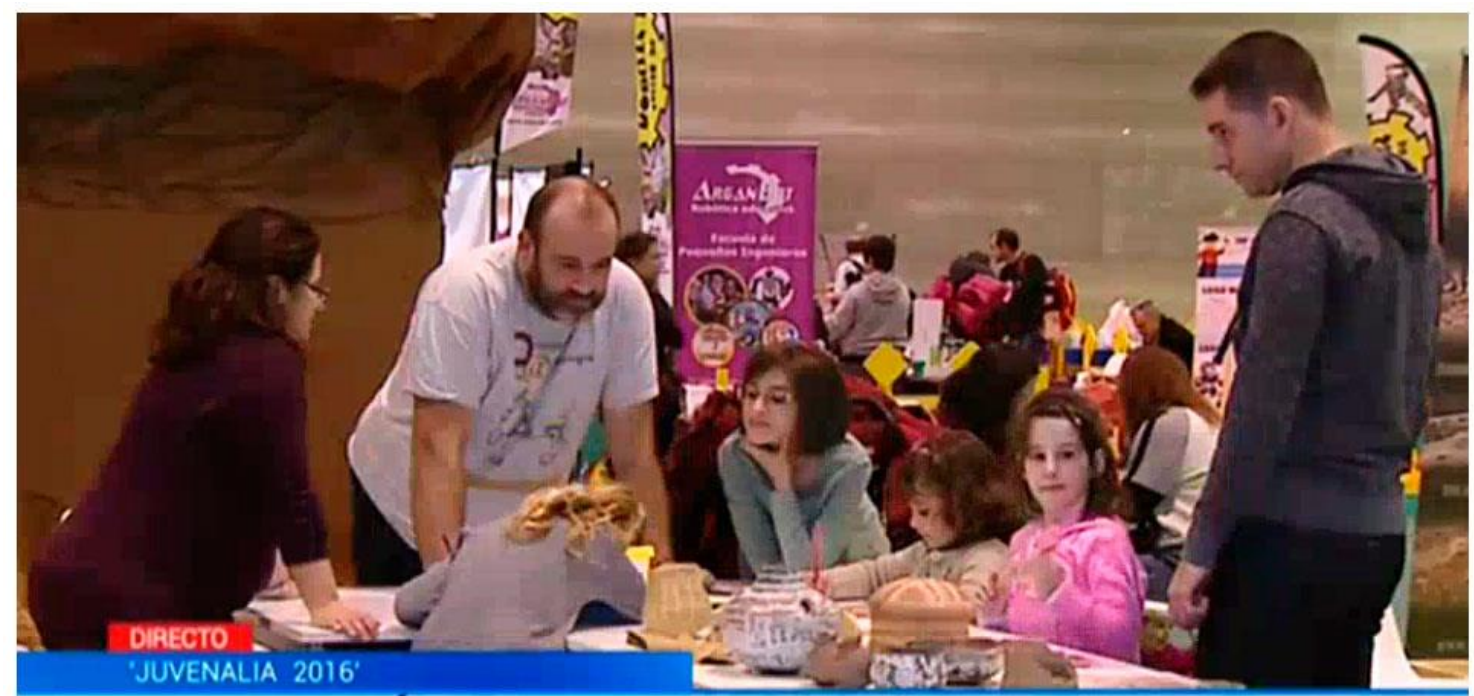

\section{HASTA EL PRÓXIMO DOMINGO SE CELEBRA EN IFEMA EL SALON DE OCIO INFANTIL Y JUVENIL}

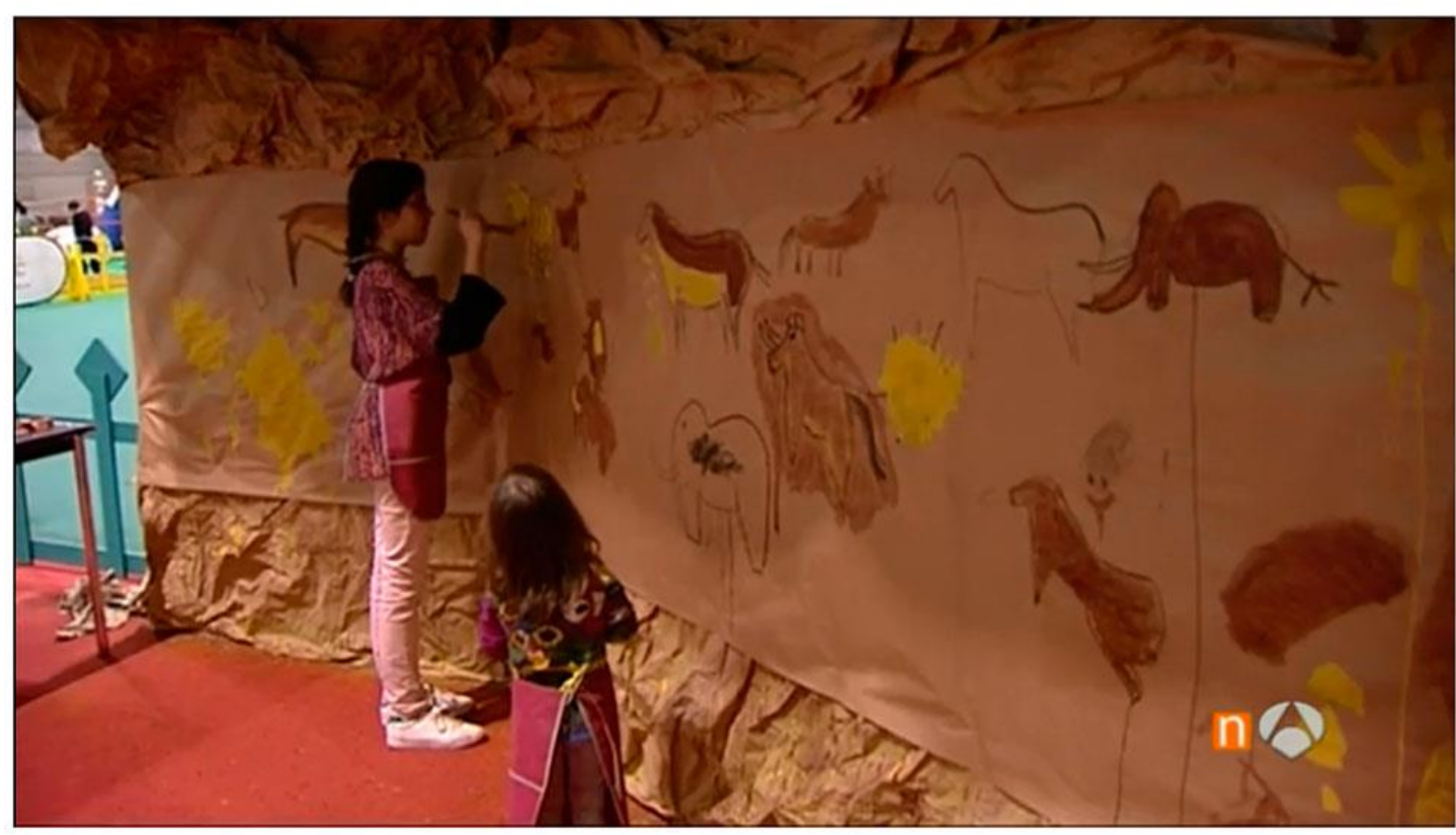

Figura 5: Cobertura informativa de los talleres arqueológicos de Juvenalia en diferentes medios de comunicación. En la imagen superior, informativos de la 1 de TVE; en la imagen inferior, Antena 3.

\section{Conclusiones}

Participar en un evento de la importancia y repercusión de Juvenalia ha sido una oportunidad única para reflexionar y aprender sobre la difusión de la Arqueología en eventos masivos.

Como aspectos positivos hay que destacar la repercusión de unos sencillos talleres ante un público muy numeroso que acudía en circunstancias a priori poco propicias para el aprendizaje reflexivo. La divulgación de la arqueología por parte de profesionales de este campo es otro aspecto que se debe valorar ya que somos capaces de detectar preconceptos erróneos, trabajar para sustituirlos por los correctos y actualizar la información a los últimos avances de la ciencia arqueológica. Además, propugnamos 
una divulgación que procede directamente de hallazgos y datos objetivos, obtenidos con metodología arqueológica.

La Arqueología es un eje transversal ideal para trabajar contenidos de todas las asignaturas del currículo escolar de educación primaria y secundaria. Cualquier aspecto del mundo actual puede tener un equivalente arqueológico con el que pueden enseñarse conceptos o procedimientos de otras disciplinas.

Las actividades reflexivas o que requieren atención y paciencia deben ser fomentadas en las ofertas de ocio infantil ya que promueven valores que se están perdiendo en el tipo de sociedad actual y que son necesarios para el desarrollo personal de los individuos, como el retardo en la recompensa (p. ej. Aguilar et al. 2013; Mischel et al. 1988). Las condiciones de masificación, ruido excesivo, constantes estímulos y la presencia de los padres, que parecía que iban a llevar a los talleres a un fracaso o a una práctica vacía de contenido, nos han servido para estudiar las adaptaciones necesarias para convertirlos en un éxito.

Creemos que tanto pedagógicamente, como a nivel de impacto, nuestra participación en Juvenalia 2016 y 2017 puede calificarse de muy exitosa y enriquecedora. Abogamos por la variedad en la oferta de ocio infantil incluyendo actividades culturales y científicas y no solamente deportivas o lúdicas. La sensación de haber sido capaz de hacer algo nuevo y de aprender algo es constructivo, gratificante y debe fomentarse también para ser practicado fuera del aula como parte de nuestro ocio.

\section{Agradecimientos}

Queremos agradecer a los organizadores de Juvenalia y a la empresa Pausanias Viajes Arqueológicos y Culturales (Jesús, Mateo y Víctor) la oportunidad, su confianza y facilidades para la realización de estas actividades. También queremos agradecer a todos nuestros colaboradores por su inestimable ayuda durante estas dos ediciones: Víctor Lamas, Paloma de la Sota, Carlos Pérez y Candela Fernández.

NC disfruta de un contrato postdoctoral dentro del Programa de Ayudas a la Atracción del Talento Investigador REF 2017-T2/HUM-3488 financiado por la Comunidad de Madrid.

\section{Bibliografía}

AGUILAR, E.; RUBIO, I. y VIÑALS, A. (2013): "El ocio digital como recurso para el aprendizaje, la socialización y la generación de capital social". Revista de Sociología de la Educación-RASE, 6(2): 196-209.

ARAGALL, A. (1987): “Actividades didácticas del área de difusión y pedagogía de la Societat Catalana d'Arqueologia". Pact News, 17: 37-39.

AUSUBEL, D. P. (2002). Adquisición y retención del conocimiento. Una perspectiva cognitiva. Ed. Paidós. Barcelona

AUSUBEL, D. P., NOVAK, J. y HANESIAN, H. (1983). Psicología educativa. Un punto de vista cognoscitivo. México. Editorial Trillas. 
BAENA, J.; TORRES, C. y PALOMO, A. (2019): “¿Seguimos jugando cuando hablamos de arqueología experimental?". Boletín de Arqueología Experimental, 13: $1-8$.

BARRERO, N.; CANALS, A.; MORCILlO, A. y PEÑA, L. (2011): "La feria de la Prehistoria en Cáceres (España): una propuesta didáctica". En A. MORGADO; J. BAENA y D. GARCÍA (eds.). La investigación experimental aplicada a la Arqueología: 483-488. Ronda: Universidad de Granada.

CARRILlO, M.; PADILlA, J.; ROSERO, T. y VILLAGÓMEZ, M. S. (2009): "La motivación y el aprendizaje". Alteridad, 4(1): 20-33.

CASTAÑEDA, N. y GONZÁLEZ, J. (2000): "Reproducción de estructuras de hábitat prehistóricos en la Flor de Maig. Una contrastación experimental del registro arqueológico para la didáctica de la Prehistoria". Boletín de arqueología experimental, 3: 18-24.

CHALMIN, E.; MENU, M. y VIGNAUD, C. (2003): «Analysis of rock art painting and technology of Palaeolithic painters". Measurement Science and Technology, 14(9): 1590.

CSIKSZENTMIHALYI, M. (1997): Flow and the psychology of discovery and invention. Nueva York: Harper Perennial.

COSTA, M. F. y DORRÍO, B. V. (2010): "Actividades manipulativas como herramienta didáctica en la educación científico-tecnológica". Revista Eureka sobre enseñanza y divulgación de las Ciencias, 2010: 462-472.

DIBUJANTES DE ARQUEOLOGÍA (2016): Pintura rupestre. [Vídeo online]. Disponible en: https://www.facebook.com/dibujantesdearqueologia/videos/vl.48879169479729 3/1466195573409067/?type=1 [Consulta: 09-06-2020]

DIBUJANTES DE ARQUEOLOGÍA (2017): Talleres de Arqueología en Juvenalia 2016 [Vídeo online]. Disponible en: https://youtu.be/a5osXccECWY [Consulta: 09-06-2020]

EFE (2009): "Madrid se queda sin Juvenalia y Expo Ocio". El País, 30 de octubre. Disponible en: https://elpais.com/economia/2009/10/30/actualidad/1256891580_850215.html [Consulta: 09-06-2020].

EL PAÍS (1980): "Juvenalia 80, nueve horas diarias para divertirse durante las Navidades". El País, 24 de diciembre. Disponible en: https://elpais.com/diario/1980/12/24/madrid/346508657_850215.html [Consulta: 09-06-2020]

EL PAÍS (1987): "Medio millón de personas visitaron Juvenalia". El País, 28 de diciembre. en: https://elpais.com/diario/1987/12/28/madrid/567692655_850215.html [Consulta: 09-06-2020] 
EUROPEAN COMMISSION. DIRECTORATE-GENERAL XXII; YOUTH. (1995): White Paper on Education and Training, Teaching and Learning, Towards the Learning Society.

GALINDO, C. (2000): "Juvenalia 2000 abre sus 30.000 metros cuadrados a casi medio millón de niños". $A B C, 21$ de diciembre. Disponible en: http://hemeroteca.abc.es/nav/Navigate.exe/hemeroteca/madrid/abc/2000/12/21/1 00.html [Consulta: 09-06-2020]

GARCÍA-MARÍN， P. y FERNÁNDEZ-LÓPEZ， N. (2020): “Asociación de la competencia en las habilidades motrices básicas con las actividades físicodeportivas extracurriculares y el índice de masa corporal en preescolares”. Retos, 38: 33-39.

GATT, S. (2003): “Constructivism: An Effective Theory of Learning”. En S. GATT y Y. VELLA (eds.). Constructivist Teaching in Primary School: Examples in Social Studies, Science, Mathematics, Design and Technology and ICT. Malta: Agenda Publishers

GIL, A.; IZQUIERDO, Mª I.; PÉREZ, C. y FIÉRREZ, S. (1994): “Arqueología en la Enseñanza. El Taller de Arqueología del I.F.P Misericordia, Valencia". Revista de Arqueología, 159: 6-11.

GÓMEZ-BAEZA, R. (1988): “Cartas al director: Juvenalia”. El País, 21 de diciembre. Disponible en: https://elpais.com/diario/1988/12/21/opinion/598662007_850215.html [Consulta: 09-06-2020].

GONZÁLEZ-MARCÉN, P. (ed.) (1996): Actes del I Seminari Arqueologia $i$ Ensenyament. Treballs d'Arqueologia, 4. Bellaterra: Servei de Publicacions de l'UAB.

GONZÁLEZ-MARCÉN, P. (ed.) (1998): Actes del II Seminari Arqueologia $i$ Ensenyament. Treballs d'Arqueologia, 5. Bellaterra: Servei de Publicacions de l'UAB.

GONZÁLEZ-MARCÉN, P.; CASTAÑEDA, N.; ARMENTANO, N.; BARAHONA, M. y GONZÁLEZ, J. (1998): "La recerca a l'abast: l'experiència del parc arqueològic del Patronat Flor de Maig”, En P. GONZÁLEZ-MARCÉN (ed.). Actes del II Seminari Arqueologia $i$ Ensenyament, pp. 65-84. Treballs d'Arqueologia, 5, Bellaterra: Servei de Publicacions de l'UAB.

HERNÁNDEZ, Ma . C. (2019): “Una experiencia educativa televisada en España entre la década de los 60 y los 80. El programa Misión Rescate”. Historia de la Educación, 38: 311-325.

JARDÓN, P. y SOLER, B. (1994): “Taller d'Arqueologia Experimental. Els nostres avantpassats. Els caçadors prehistórics". Recerques del Museu d'Alcoi, 3.

KIM, S.; SONG, K.; LOCKEE, B. y BURTON, J. (2018): Gamification in Learning and Education. Enjoy Learning Like Gaming. Springer. 
LLULL, J. (2011). "Pedagogía del ocio. Coordinadores de tiempo libre" [Recurso Electrónico]. https://eala.files.wordpress.com/2011/02/pedagogc3ada-del-ocio.pdf

[Consultado el 10/11/2020]

MĂRGĂRIT, M. (2016): "Testing the endurance of prehistoric adornments: raw materials from the aquatic environment". Journal of Archaeological Science, 70: 66-81.

MISCHEL, W.; SHODA, Y.; PEAKE, P. K. (1988): "The nature of adolescent competencies predicted by preschool delay of gratification". Journal of personality and social psychology, 54 (4): 687.

MR. CONTRAINTUITIVO (2019) [Blog]: "El inmenso peligro de la adicción a la recompensa inmediata". 30 de enero. Disponible en: https://mrcontraintuitivo.com/peligros-recompensa-inmediata/ [Consultado el $10 / 11 / 2020]$.

MORGADO, A. y BAENA, J. (2011) “Experimentación, Arqueología experimental y experiencia del pasado en la Arqueología actual”. En A. MORGADO, J. BAENA y D. GARCÍA (eds.). La Investigación Experimental aplicada a la Arqueología, pp. 21-28. Ronda: Universidad de Granada.

O'BRIEN, L.; ALBERT, D.; CHEIN, J. y STEINBERG, L. (2011): “Adolescents prefer more immediate rewards when in the presence of their peers". Journal of Research on adolescence, 21(4), 747-753.

PALEOAPRENDE (2013) [Blog] Disponible en: http://paleoaprede.blogspot.com/ [Consultado el 10/11/2020]

PONS, R. M. y SERRANO, J. M. (2011): "La adquisición del conocimiento: una perspectiva cognitiva en el dominio de las matemáticas". Educatio Siglo XXI, 29(2): 117-138.

POU, J.; SANMARTÍ, J. y SANTACANA, J. (1995): "La reconstucció del poblat ibèric d'Alorda Park o de les Teixoneres (Calafell, Baix Penedès)". Tribuna d'Arqueologia, 1993-1994.

POZO, Á. del (1990): "La ciudad, convertida en una gran oferta de festejos para los niños". $A B C, \quad 23$ de diciembre. Disponible en: http://hemeroteca.abc.es/nav/Navigate.exe/hemeroteca/madrid/abc/1990/12/23/0 53.html [Consulta 09-06-2020]

PROYECTO ARQUEO (2015) [Sitio Web] Disponible en: https://proyectoarqueo.com/ [Consulta 10-11-2020]

SHERNOFF, D. J. y HOOGSTRA, L. (2001): "Continuing motivation beyond the high school classroom". New Directions for Child and Adolescent Development, 2001(93): 73-88.

Tiza, Educación y Sociedad: Revista de Juvenalia (1984). Número 1, 1 de mayo. Madrid: IFEMA. 
VILLAR, R. y VÁZQUEZ, B. (2006): “Aproximación a la arqueología: un ejemplo de interpretación del hecho científico". Íber: Didáctica de las ciencias sociales, geografia e historia, 48: 115-125. 